\title{
MASTERCLASS
}

\section{An unexpected cause of muscle pain in diabetes}

\author{
L Silberstein, K E Britton, F P Marsh, M J Raftery, D D’Cruz
}

St Bartholomew's and the Royal London School of Medicine

UK

L Silberstein

K E Britton

F P Marsh

M J Raftery

D D'Cruz

Correspondence to:

Dr D D'Cruz, The Lupus

Institute, St Thomas's

Hospital, Lambeth Palace

UK

Accepted 20 October 2000

\begin{abstract}
Diabetic muscle infarction is a rare condition which may present to a rheumatologist. It was first reported in 1965. Two illustrative cases are described here and the mechanisms of pathogenesis discussed. Analysis of the published data, results of the muscle biopsies, and a technetium-99m sestamibi scan suggest that the condition, which occurs against a background of diabetic microangiopathy, background of diabetic microangiopathy, can be triggered by an ischaemic event
and causes extensive muscle necrosis and causes extensive muscle necrosis
through hypoxia-reperfusion injury and compartment syndrome.

(Ann Rheum Dis 2001;60:310-312)
\end{abstract}

Diabetic muscle infarction is a rare cause of acute severe muscle pain in patients with diabetes mellitus. The differential diagnosis includes focal or systemic myositis, localised includes focal or systemic myositis, localised
abscess, haematoma, deep venous thrombosis, abscess, haematoma, deep venous thrombosis, osteomyelitis, and a muscle tumour (sarcoma or lymphoma). We describe two illustrative patients and discuss the investigations and possible pathogenesis of this condition.

Case 1

A 46 year old West Indian woman with a 14 year history of type II diabetes mellitus complicated by diabetic nephropathy and proliferative retinopathy presented to a local liferative retinopathy presented to a local
hospital with a painful swollen left leg after an insulin injection into the left femoral canal area. Deep venous thrombosis was excluded by Doppler ultrasound, and a computed tomography (CT) scan showed swelling of the left vastus medialis muscle. The erythrocyte sedimentation rate (ESR) was raised at $130 \mathrm{~mm} / 1 \mathrm{st} \mathrm{h}$, tation rate (ESR) was raised at $130 \mathrm{~mm} / 1 \mathrm{st} \mathrm{h}$, (ANCA), antinuclear antibodies (ANA), and

She was given intravenous albumin and diuret. swelling of the leg was prescribed, the symptoms gradually improved. She did, however, develop contractures in the thigh muscles requiring physiotherapy. At the time of discharge she could walk with a stick.

She presented again five months later with a one week history of painful swelling of the pposite thigh. On this occasion, there were no precipitating factors. The medial compartment of the right thigh was markedly swollen. Ther was no neutrophilia or CK rise. A CT scan showed generalised swelling of the anterior and medial groups of muscle and connective tissue from the pelvis distally.

The patient underwent surgical debridement of the mass. The sartorius muscle was necrotic and was excised. Swollen but viable muscles in the adductor and quadriceps compartment were noted. Histological examination showed widespread necrosis. The vessels had lumenal stenosis and calcification consistent with longstanding diabetes.

In view of the worsening renal failure, peritoneal dialysis was started. She remained well for the next four years, but her condition later deteriorated and she died from a complication of peritoneal dialysis.

\section{Case 2}

A 55 year old Afro-Caribbean man with a 24 year history of type II diabetes complicated by peripheral neuropathy, proliferative retinopathy, and nephropathy requiring continuous ambulatory peritoneal dialysis (CAPD) presented to his local hospital with a tender swellin on his left upper lateral thigh. Serial blood ulins, ANA, antidsDNA, and ANCA were all negative. A biopsy showed striated muscle exhibiting infarction and infiltration by neutrophils. The arteries showed lumenal thrombosis and organisation, and small arterioles showed fibrinoid necrosis. Stains for bacteria and fungi were negative. The patient was given fusidic acid
flucloxacillin and gradually improved.

Two months later he re-presented with painful well defined swelling in the left thigh. $C$ reactive protein (CRP) on admission was 60 $\mathrm{mg} / \mathrm{l}$ (reference range $0-10 \mathrm{mg} / \mathrm{l}$ ) and rose to $105 \mathrm{mg} / \mathrm{l}$ one week later. He was given intrakinase (CK) $202 \mathrm{IU} / 1$ (reference range 0-170 venous flucloxacillin and ciprofloxacin for four
$\mathrm{IU} / \mathrm{l}$ ), ESR $97 \mathrm{~mm} / 1 \mathrm{st} \mathrm{h}$, and albumin $16 \mathrm{~g} / \mathrm{l}$. A $\begin{array}{ll}\text { kinase (CK) } 202 \mathrm{IU} / 1 \text { (reference range } 0-170 & \text { venous flucloxacillin and ciprofloxacin for four } \\ \mathrm{IU} / \mathrm{l}), \text { ESR } 97 \mathrm{~mm} / 1 \mathrm{st} \text { h, and albumin } 16 \mathrm{~g} / \mathrm{l} \text {. A } & \text { weeks, but failed to improve and the entire } \\ \text { renal biopsy confirmed diabetic nephropathy. } & \text { mass was excised. Histological findings were }\end{array}$ 


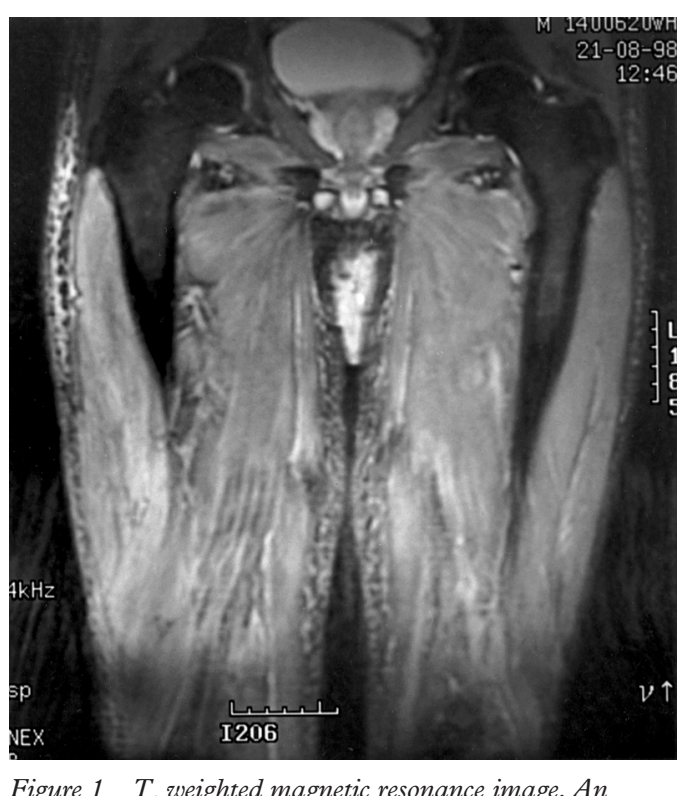

Figure $1 \quad T_{2}$ weighted magnetic resonance image. An can be seen.

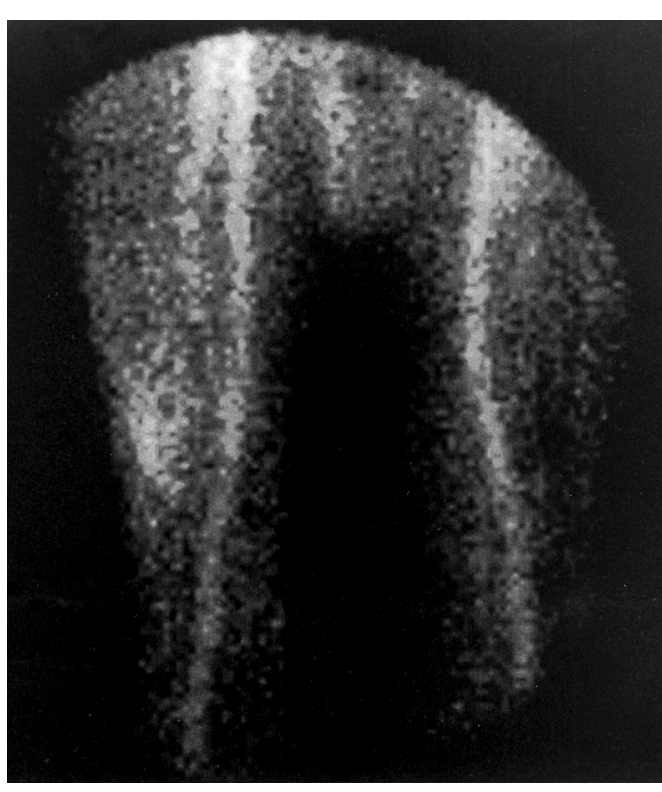

Figure 2 Dynamic ${ }^{9}$ Tc-sestamibi scan. Increased tracer uptake at the site of the lesion in the right thigh is seen.

similar to the previous biopsy, but arteriolar fibrinoid necrosis was more marked.

Ten months later the patient was admitted with pain and swelling of the opposite thigh, which had developed over a few weeks. O examination he had a large discrete swelling on the lateral aspect of the right thigh which was warm and exquisitely tender. He had a mild neutrophilia, markedly raised CRP of $132 \mathrm{mg} / \mathrm{l}$, but normal CK. A plain $x$ ray examination of the right hip was normal, apart from widespread vascular calcification. Doppler ultrasound showed no evidence of a deep vein thrombosis. A magnetic resonance imaging (MRI) scan of the thighs showed an area of increased signal density in the right vastus lateralis compartment consistent with oedema of the muscle (fig 1). He was treated with intravenous antibiotics and analgesia. His symptoms gradually improved, he no longer required opiates, and could walk with a stick. A technetium $(\mathrm{Tc})$ labelled bone scan performed two weeks after discharge showed increased blood flow to the affected area of the muscle with slightly increased tracer uptake in the soft tissues and normal uptake in the skeletal system.

The patient was readmitted a month later with worsening pain in the right thigh. Serial $\mathrm{CK}$ measurements remained normal. He was given a small dose of steroids and his condition improved. A ${ }^{99 m}$ Tc-sestamibi scan showed increased vascularity at the lesion in the right thigh, seen on the bone scan, and the presence of living muscle (fig 2).

Over the next three months he was admitted several times with infection of the fascial spaces of the hand and CAPD peritonitis; he died from the complications of diabetes.

\section{Discussion}

Diabetic muscle infarction is a rare complication of diabetes, which should be suspected in any diabetic subject with atypical severe muscular pain. It was first described in 1965 by Angerall and Stener as a "tumoriform focal muscular degeneration" 1 and since then has been reported in a total of 86 patients. ${ }^{1-33}$ Sixty five patients had type I diabetes, 19 patients cotien ad type II diabetes, and in two patients the type was not specified. The male/female ratio was almost equal (44/42), with an age range of 19-81 years. Most patients had longstanding diabetes and extensive end-organ damage due to microvascular disease.

The condition presents as an atraumatic swelling of the limb, commonly the thigh. The onset of pain is usually gradual, but can be sudden. The swelling is exquisitely tender. It resolves within a few weeks, but frequently recurs. The white cell count and the level of CK are normal or slightly raised. Muscle biopsy typically shows large confluent areas of muscle necrosis and oedema. ${ }^{2}$ The best imaging results are with $\mathrm{T}_{2}$ weighted MRI scans, which have a fairly characteristic, but nonspecific appearance showing the absence of a specific appearance showing the absence of a
discrete mass and increased signal within the discrete mass an
affected muscle.

The differential diagnosis includes a muscle tumour (sarcoma or lymphoma), localised abscess, haematoma, focal or systemic myosinous thrombosis, and The management should include bed rest analgesia, tight metabolic control, and physiotherapy. ${ }^{2}$

Various mechanisms of pathogenesis have been proposed. Earlier reports focused on diabetic microangiopathy, atheromatosis, ${ }^{1}$ and embolisation of atheromatous material from lcerated aortic plaques as the causes of muscle infarction ${ }^{5}$ In the presence of diabetic microvascular disease, a thromboembolic event is vascular disease, a thromboembolic event is
more likely to lead to infarction because of mpaired collateral circulation. ${ }^{6}$ However, later reports showed that only a minority of cases had a vascular occlusion which would correspond to the extent of muscle necrosis. The spond to the 
not cause infarction but leads to it by producing muscle oedema which increases the pressure within a fascial compartment and causes further ischaemia.?

We suggest that hypoxia-reperfusion injury may have an important role in the pathogenesis of diabetic muscle infarction. The likely sequence of events leading to muscle necrosis is as follows. Compartment syndrome, precipitated by a small thrombotic/embolic event or intramuscular insulin injection, produces ischaemic muscle damage. This leads to a potent inflammatory response, hyperaemia, and reperinflammatory response, hyperaemia, and reperfusion with generation of reactive oxygen species causing further muscle damage, both directly and through worsening of the compartment syndrome due to muscle oedema. Thus there is a "vicious circle" which eventually results in extensive muscle necrosis. It is of note that a Tc labelled bone scan in case 2 confirmed the presence of hyperaemia, which was consistent with the findings of other investigators. ${ }^{8}$ Although a ${ }^{99 \mathrm{~m}}$ Tc-sestamibi scan tigators. $^{89}$ Although a ${ }^{99 m}$ Tc-sestamibi scan
showed the presence of a living muscle at the showed the presence of a living muscle at the
site of the injury, it is possible that the "vicious site of the injury, it is possible that the "vicious circle" was disrupted and the images were occurred. Interestingly, Jawed et al have shown that cyclical hypoxia-reperfusion injury is responsible for synovial damage in chronic inflammatory arthropathies as they are characterised by a rise in the intra-articular pressure above the capillary perfusion pressure.

The clinical features of our two patients closely resemble those of previously reported cases. Both of them had longstanding diabetes with retinopathy, neuropathy, and nephropathy requiring CAPD. They both presented with tequiring CAPD. They both presented with tender recurrent swelling affecting the muscles
of the legs and improved with supportive treatof the legs and improved with supportive treatment, though steroids may have contributed to the recovery in the second patient. The diagnosis was confirmed by a muscle biopsy, except for the last recurrence of the disease in the second patient, when the procedure was not performed in view of the similar clinical presentation and characteristic MRI findings.

Diabetic muscle infarction is a rare conditio that has become more frequently recognised in the past few years. It should be suspected in a patient with a longstanding diabetes who presents with a painful swollen limb. MRI is the best imaging modality, but early core-needle or open muscle biopsy at first presentation is essential to establish the diagnosis.

1 Angerall $\mathrm{L}$, Stener B. Tumoriform focal muscular degenera-
tion in two diabetic patients. Diabetologia 1965;1:39-42. 2 Umpierezz GE, Stiles RG, Kleinbart J, Krendel DA, WWatts 50.
Van Slyke MA, Ostrov BE. MRI evaluation of diabetic mus-
4 Jelinek JS, Murphey MD, Aboulafia AJ, Dussault RG, Kaplan PA, Snearly WN. Muscle infarction in patients with 211:241-7.

5 Banker BQ, Chester CS. Infarction of thigh muscle in the

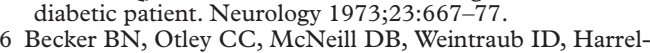
son JM. Microangiopathic ischemic myopathy of semmembranosus muscle in patient with diabetes mellitus. Diabetes Care 1992;15:586-7.

Chester CS, Banker BQ. Focal infarction of muscle in diabetics. Diabetes Care 1986;9:623-20. logical aspects of diabetic muscle infarction. Ration approach to diagnosis and treatment. J Bone Joint Surg Br

KF. Diabetic muscle infarction. J South 0 Jawed S, Gaffney K, Blake DR. Intra-articular pressure pro-
and file of the knee joint in a spectrum of in

Sagar M, Bowerfind WM, Wigley FM. A man with diabetes and a swollen leg. Lancet 1999;353:116.

A, Nicholls AJ. Diabetic muscle continuous ambulatory peritoneal dialysis. Nephrol Dia Transplant 1998;13:2377-9.

3 Reich S, Weimer SN, Chester S, Ruff R. Clinical and radiocle infarction in the

Skeletal muscle infarction in diabetes: MR findings. Comput Assist Tomogr 1993;17:986-8.

( 作 16 Barohn RJ, Kissel JT. Case-of-the-month: painful thig mass in a young woman: diabetic muscle inf preve Muscle

Hinton A, Heinrich SD, Craver R. Idiopathic diabetic mus-
cular infarction: the role of ultrasound, MRI and biopsy. Orthopaedics 1993;16:623-5.

imaging avoids the need for biopsy. Muscle Nerve imaging avoids
1995;18:129-30.

19 Weissman J. Diabetic muscle infarction. Radiographics

muscle infarction: radiologic evaluation. Skeletal Radio 1 Barton KL, Palmer BF. Bilateral infarction of the vastus la eralis muscle in a diabetic patient: a case report and revie 2 Boluda B, Mesa J, Obiols G, Simo R. Focal muscle infarction in a diabetic. Diabete Metab 1989;15:269-70. Bodn RA, Yonger DS, Rosolija G. Diabjic:

J, Blalock JC, Kasin JV. Infarction of lopathy. South Med J 1986;79:1595.

(a) infarction: report of two cases. Skeletal Van de Berg B, Malchem J, Puttemans T, Vandeleene Lagneau G, Maldague B. Jdiopathic muscular infarction a diabetic patient. Skeletal Radiol 1996;25:183-5.
Bjornskov EK. Carry . Diabetic muscle infarction: a new perspective on pathogeesis and management. Neuromuscul Disord 1995;5:39-45. Stadnick M, Rooney M. Idiopathic necrosis of skelet muscle in patients who have diabetes. J Bone Joint Surg Am

1998;80:262-7.
Heureux F, Nisolle JF, Delgrange E, Donckier J. Diabetic muscle infarction: a difficult diagnosis suggested by

$621-2$
30 Taira $M$, Komia I, Taira I, Arakawa T, Hokama S,
Nagasawa Y, et al. A case of diabetic muscle infarction in Nagasawa Y, et al. A case of diabetic

1 Penglis PS, Scott G, Cleland LG. Diabetic muscle infarction presenting as a knee effusion. Semin Arthritis Rheu

1999,28:421-2.

Lauro GR, Kissel JT, Simon SR. Idiopathic muscular
infarction in diabetic patient. J Bone Joint Surg Am 1991, 73:301-4.

33 Rocca PV, Alloway JA, Nashel DJ. Diabetic muscl 